\title{
Bacanora: Una bebida regional con denominación de origen que impulsa el turismo de reuniones
}

\section{Bacanora: A regional drink with a designation of origin that promotes meeting tourism}

\author{
Dra. Yesenia Clark Mendivil ${ }^{1}$, Dra. Irma Guadalupe Esparza García ${ }^{2}$, Dra. \\ Martha del Pilar Rodríguez García ${ }^{3}$
}

${ }^{1}$ yessenia.clark@itson.edu.mx http://orcid.org/0000-0003-2032-7433 / Instituto

\section{Tecnológico de Sonora}

2iesparza@itson.edu.mx https://orcid.org/0000-0002-3715-076. / Instituto

\section{Tecnológico de Sonora}

${ }^{3}$ martha.rodriguezgc@uanl.edu.mx https://orcid.org/0000-0001-8262-958X.

\section{/Universidad Autónoma de Nuevo León}

\section{DOI https://doi.org/10.46589/rdiasf.vi35.393}

Recibido 26 de mayo 2021.

Aceptado 20 de junio 2021

Publicado 30 de junio de 2021

\section{Resumen}

México tiene 17 denominación de origen regulados por el instituto mexicano de la propiedad industrial ([IMPI]), mismos que se encuentran distribuidos a lo largo y ancho de todo el territorio mexicano: El estado de Sonora tiene una denominación de origen el "Bacanora" que es una bebida alcohólica regional que se produce a partir de la destilación del agave angustifolia haw por su nombre científico.

La oferta de productos y servicios en la industria del turismo del sur de Sonora es escasa, la necesidad de investigación, de formación, de mercadeo, de intercambio de ideas entre especialistas sobre el tema del turismo y considerando la oportunidad que el bacanora representa para el desarrollo de esta como la única bebida emblemática y con denominación de origen es una de las problemáticas a abordar en esta investigación. 
La metodología empleada tiene un enfoque mixto con alcance exploratorio y descriptivo del fenómeno de la industria del Bacanora y del turismo de reuniones específicamente en eventos especializados como ferias y exposiciones de productos o servicios relacionados al tema del Bacanora que concurren en un área específica con el objetivo de promover negocios.

Los resultados que se presentan muestran de 410 sujetos participantes como asistentes al evento de Bacanora fest, destacando en su mayoría la presencia local, ya que se busca conocer las características de la población que asiste; el hallazgo encontrado muestra evidencia que las actividades de negocio, culturales, espectáculos y académicos fueron los más valorados por los asistentes, así como el impacto económico en la derrama económica que genera el desarrollo de estos festivales.

Palabras claves: Bacanora, denominación de origen, Turismo de Reuniones

\begin{abstract}
Mexico has 17 denominations of origin regulated by the Mexican institute of industrial property ([IMPI]), which are distributed throughout the entire Mexican territory: The state of Sonora has a denomination of origin of "Bacanora" which is a regional alcoholic beverage that is produced from the distillation of agave angustifolia haw by its scientific name.
\end{abstract}

The offer of products and services in the tourism industry in southern Sonora is scarce, the need for research, training, marketing, exchange of ideas between specialists on the subject of tourism and considering the opportunity that the bacanora represents for the development of this as the only emblematic drink with a designation of origin is one of the problems to be addressed in this research.

The methodology used has a mixed approach with an exploratory and descriptive scope of the phenomenon of the Bacanora industry and meeting tourism specifically in specialized events such as fairs and exhibitions of products or services related to the subject of Bacanora that take place in a specific area with the objective to promote business. 
The results that are presented show 410 participating subjects as attendees of the Bacanora fest event, most of them highlighting the local presence, since it seeks to know the characteristics of the population that attends; The findings found show evidence that business, cultural, entertainment and academic activities were the most valued by the attendees, as well as the economic impact on the economic spillover generated by the development of these festivals.

Keywords: Bacanora, denomination of origin, meeting tourism

\section{Introducción}

El origen de las denominaciones de origen tuvo sus inicios en el contexto internacional en el "Arreglo de Lisboa" relativo a la Protección de las Denominaciones de Origen y su Registro Internacional desde el 31 de octubre de 1958, a la fecha existen 35 países del mundo que pertenecen a este acuerdo; dentro de los cuales se descatan 6 países latinoamericanos e.g. (Costa Rica, Cuba, México, Nicaragua, Perú y República Dominicana) (Organización Mundial de la propiedad intelectual ([OMPI])2010).

En éste arreglo establece en su artículo primero que los países a los cuales se aplica se constituyen en unión particular dentro del marco de la unión para la protección de la propiedad industrial.

Así mismo, "Se comprometen a proteger en sus territorios, según los términos del presente Arreglo, las denominaciones de origen de los productos de los otros países de la Unión particular, reconocidas y protegidas como tales en el país de origen y registradas en la Oficina Internacional de la Propiedad Intelectual ([OMPI]) (llamada en lo sucesivo la "Oficina Internacional" o la "Oficina”) a la que se hace referencia en el Convenio".

Es así que la Organización Mundial de la propiedad intelectual (OMPI) es el foro mundial en lo referente a servicios, políticas, cooperación e información en materia de propiedad intelectual. Cuya misión es llevar la iniciativa en el desarrollo de un sistema internacional de propiedad intelectual equilibrado y eficaz, que permita la innovación y la creatividad en beneficio de todos. 
Considerando lo anterior y haciendo énfasis en el contexto mexicano, en México existe el Instituto Mexicano de la Propiedad Industrial ([IMPI]) que es un Organismo público descentralizado con personalidad jurídica y patrimonio propio y con la autoridad legal para administrar el sistema de propiedad industrial en nuestro país.

Este mismo instituto establece que, los productos originarios de una región, localidad o lugar del país, cuando su calidad, u otra característica se asocie a su origen geográfico son protegidos por el sistema de propiedad industrial cuya elaboración se hace incluyendo métodos tradicionales, relacionados a las costumbres de zonas geográficas delimitadas, cuya principal característica es que los productos elaborados incluyen una importante carga histórica y cultural (Instituto Mexicano de la Propiedad Industrial, 2018).

La denominación de origen y la indicación geográfica son bienes de dominio del poder público de la Federación y se reconocen a través de una declaratoria de protección emitida por el IMPI. Por medio de las Denominaciones de Origen y las indicaciones Geográficas, se protegen productos agroalimentarios y manufacturados, tales como las artesanías y las bebidas típicas.

México tiene 17 denominación de origen regulados por el IMPI, mismos que se encuentran distribuidos a lo largo y ancho de todo el territorio mexicano como se muestra en la Tabla 1 y que son regulador por el IMPI. 
Tabla 1.

Denominaciones de origen en México

\begin{tabular}{|c|c|c|c|}
\hline $\begin{array}{c}\text { Denominación de } \\
\text { origen }\end{array}$ & Categoría & Producto & $\begin{array}{c}\text { Estados de la república mexicana } \\
\text { protegidos para producir }\end{array}$ \\
\hline 1. Tequila & Bebida típica & $\begin{array}{l}\text { Destilado de } \\
\text { Agave }\end{array}$ & $\begin{array}{l}\text { Jalisco, Michoacán, Tamaulipas, } \\
\text { Nayarit y Guanajuato }\end{array}$ \\
\hline 2. Mezcal & Bebida típica & $\begin{array}{l}\text { Destilado de } \\
\text { Agave }\end{array}$ & $\begin{array}{l}10 \text { estados: Durango, Guanajuato, } \\
\text { Guerrero, Michoacán, San Luis } \\
\text { Potosí, Puebla, Zacatecas, } \\
\text { Tamaulipas, Oaxaca y } \\
\text { Aguascalientes }\end{array}$ \\
\hline 3. Bacanora & Bebida típica & $\begin{array}{l}\text { Destilado de } \\
\text { Agave }\end{array}$ & 1 Estado: Sonora \\
\hline 4. Sotol & Bebida típica & $\begin{array}{l}\text { Destilado de } \\
\text { Agave }\end{array}$ & $\begin{array}{l}3 \text { estados: Chihuahua, Durango y } \\
\text { Coahuila }\end{array}$ \\
\hline 5. Raicilla & Bebida típica & $\begin{array}{l}\text { Destilado de } \\
\text { Agave }\end{array}$ & 1 Estado: Jalisco \\
\hline 6. Charanda & Bebida típica & $\begin{array}{l}\text { Destilado de } \\
\text { Caña de azúcar }\end{array}$ & 1 Estado: Michoacán \\
\hline 7. Café Veracruz & Alimentos & Café & 1 Estado: Veracruz \\
\hline 8. Café Chiapas & Alimentos & Café & 1 Estado: Chiapas \\
\hline $\begin{array}{l}\text { 9. Mango Ataulfo } \\
\text { del Soconusco } \\
\text { de Chiapas }\end{array}$ & Alimentos & $\begin{array}{l}\text { Fruto del } \\
\text { mango }\end{array}$ & 1 Estado: Chiapas \\
\hline $\begin{array}{l}\text { 10. Vainilla de } \\
\text { Papantla }\end{array}$ & Alimentos & $\begin{array}{l}\text { Fruto de una } \\
\text { orquídea }\end{array}$ & 1 Estado: Veracruz \\
\hline $\begin{array}{l}\text { 11. Chile Habanero } \\
\text { de la Península } \\
\text { de Yucatán }\end{array}$ & Alimentos & Verdura: Chile & $\begin{array}{l}3 \text { Estado: Yucatán, Quintana Roo y } \\
\text { Campeche }\end{array}$ \\
\hline $\begin{array}{l}\text { 12. Arroz del } \\
\text { Estado de } \\
\text { Morelos }\end{array}$ & Alimentos & Arroz & 1 Estado: Morelos \\
\hline 13. Cacao Grijalva & Alimentos & Cacao & 1 Estado: Tabasco \\
\hline 14. Yahualica & Alimentos & $\begin{array}{l}\text { Fruto de chile } \\
\text { de árbol }\end{array}$ & 1 Estado: Jalisco \\
\hline 15. Olinalá & Artesanía & $\begin{array}{l}\text { Artesanía de } \\
\text { madera }\end{array}$ & 1 Estado: Guerrero \\
\hline 16. Talavera & Artesanía & $\begin{array}{l}\text { Loza con } \\
\text { esmalte } \\
\text { metálico }\end{array}$ & 2 Estados: Puebla y Tlaxcala \\
\hline $\begin{array}{l}\text { 17. Ámbar de } \\
\text { Chiapas }\end{array}$ & Artesanía & $\begin{array}{l}\text { Piedra } \\
\text { semipreciosa de } \\
\text { origen vegetal }\end{array}$ & 1 Estado: Chiapas \\
\hline
\end{tabular}

Fuente: Elaboración propia con información del Instituto mexicano de la propiedad intelectual (2018) 
Analizando la tabla anterior podemos observar que las protecciones de los productos en el territorio mexicano se han centrado principalmente en la categoría de alimentos o del sector agroalimentario con un mayor número de protecciones (8), seguido de las bebidas típicas (6) y después las artesanías (3).

Considerando lo anterior vamos a retomar el tema de las bebidas típicas, en México, son las bebidas las que ocupan el segundo lugar en el tema de protección de la denominación de origen, la variedad de bebidas destiladas de diferentes tipos de agaves, las cuales en la actualidad se ven impulsadas por una demanda nacional e internacional, e.g. (tequila, el mezcal, el sotol el bacanora, y recientemente incluida la raicilla), siendo representativas cada una de ellas, de alguna región diferente del país (ver tabla 1). Su diferencia se basa en el tipo de agave, planta que se utiliza como materia prima para su elaboración, así como en el proceso de elaboración (Mancillas, López 2006; Oliveras 2008).

En el estado de Sonora existe la protección de una denominación de origen de la bebida típica que es un destilado de agave denominado "Bacanora" la cual representa una oportunidad de desarrollo y crecimiento para el sector económico y social del estado y de los municipios que tienen la protección, por lo que será en tema de énfasis en esta investigación.

Según Gutiérrez (2007), el bacanora es una bebida ancestral que tiene más de 300 años de existencia, la tribu de optas que habitaban en Sonora, fueron los que le pusieron el nombre de Bacanora a la bebida después de descubrir su proceso de destilación. El bacanora únicamente se podía producir por una especie de agave que era el que abundaba en los terrenos de los indígenas que ocupan cierta región sonorense, este agave lleva por nombre científico Angustifolia Haw, al cual también se le conoce popularmente cono yaquiana o pacifica, el cual es asado o tatemado, fermentado y posteriormente destilado en un proceso muy similar a otros mezcales como el tequila, pero siendo diferente por el tipo de agave.

Así, aquellos indígenas ópatas que ocupaban el espacio actualmente conocido como el estado de Sonora, utilizaban el mezcal para fiestas y ceremonias de sus comunidades, bebida elaborada por ellos mismos y quienes más adelante se verían 
influenciados por los españoles para destilar las bebidas una vez fermentadas (Salazar, 2007).

A raíz de la crisis económica del siglo XIX, el bacanora fungía como una fuente de ingresos para la hacienda tributaria, de esta manera se inició la regularización de los alambiques con los cuales se elaboraba la bebida, imponiendo un impuesto por la venta de la bebida y por patentes de alambiques (Ulloa, 1910).

Sin embargo, en el año de 1915 la producción de la bebida presentó un problema, se decretó la prohibición absoluta para la producción, consumo y comercialización de bebidas alcohólicas de cualquier tipo en el estado de Sonora, convirtiéndose en un delito sancionado con pena de cárcel para quien lo cometiera y sus cómplices, esto organizado por el General Plutarco Elías Calles quien era el entonces gobernador del estado de Sonora, este decreto fue mejor conocido como "Ley Seca" hecho por el cual la bebida se vio perjudicada inmensamente (Salazar, 2007).

Pero la bebida ya representaba un auge comercial, al prohibir su producción no solo afectaba el desarrollo de la industria, sino afectaba las economías de muchas familias que dependían del ingreso que les generaba, era echar por la borda una tradición que se venía pasando y heredando generación tras generación y en esa época ya representaba un bien cultural. Al no acatar el mandato su producción, la bebida continuo desarrollándose clandestinamente por muchos productores de la sierra del estado, quienes se aferraron a sus culturas y tradiciones, pues representaba una fuente de ingresos para sus familias, de esta manera la bebida se vio expuesta ante un mercado informal, dentro del cual fue evolucionando, hasta llegar el año de 1992, 77 años después de su prohibición, el gobierno del estado de Sonora, inicio la formalización de la producción, comercialización y consumo de bacanora diseñando cierta normas que regularizaran la bebida (Boletín Oficial del Gobierno del Estado de Sonora, 1992).

Así mismo en el año 2000 hace apenas 21 años, que se le otorga oficialmente el nombre de la denominación de origen y producto o productos que se pretende amparar: "Bacanora", para ser aplicada a una bebida alcohólica regional del Estado de Sonora a través del Diario Oficial de la Federación, (DOF, 2000).

Esta bebida toma su nombre del Municipio de Bacanora, situado en la Sierra Centro, al este de Hermosillo, capital del Estado de Sonora. Esta bebida tradicional ha llegado a representar uno de los símbolos de identidad de los Sonorenses. No obstante, 
la zona tradicional de producción abarca un territorio más amplio, en el cual se incluyen 35 municipios.

La zona geográfica para proteger la denominación de origen, incluye municipios de las regiones Sierra Centro, Río Sonora y San Miguel; Centro; Sierra Alta, y Sierra Sur. Estos municipios son los siguientes: Bacanora, Sahuaripa, Arivechi, Soyopa, San Javier, Cumpas, Moctezuma, San Pedro de la Cueva, Tepache, Divisaderos, Granados y Huásabas, Villa Hidalgo, Bacadehuachi, Nácori Chico, Huachinera, Villa Pesqueira, Aconchi, San Felipe de Jesús, Huépac y Banámichi, Rayón, Baviácora, Opodepe, Arizpe, Rosario, Quiriego, Suaqui Grande, Onavas, Yécora, Álamos, San Miguel de Horcasitas, Ures, Mazatán y La Colorada, todos éstos comprendidos en el Estado de Sonora, el cual se caracteriza por su tradición en la elaboración de Bacanora (Diario Oficial de la Federación, (DOF, 2000)).

De tal manera que para el desarrollo de la industria ha sido necesario realizar diferentes investigaciones, para poder fomentar su desarrollo tal es el caso del Centro de Investigaciones en Alimentación y Desarrollo quien realizó un estudio sobre del desarrollo estratégico de la industria del bacanora (Núñez 2004), tal investigación destaca la necesidad de dar un lugar a todos aquellos cambios actuales que afectan o benefician de manera directa e indirecta a todos aquellos involucrados en la actividad, el desarrollo de la actividad requiere una especialización para poder adaptar el proceso tradicional sin dañar o afectar la cultura y tradición a la cual se arraiga, es de vital importancia aumentar la comercialización, desarrollo tecnológico, financiamiento, y promoción de la bebida para que esta obtenga el reconocimiento que se merece al ser ya un símbolo de identidad Sonorense.

Para poder enriquecer el desarrollo de la industria del bacanora es importante conocer los problemas y limitaciones que se presentan en la producción, la falta de materia prima, el análisis del mercado de la actividad, la promoción y difusión de lo que significa para un estado tener una denominación de origen de una bebida regional como un símbolo de identidad Sonorense.

Para el desarrollo del sector turístico en Sonora la bacanora representa un área de oportunidad, ya que como es bien conocido el turismo genera un aporte indudable a la economía, por ser una industria que genera empleos y ser un detonador del desarrollo 
local y regional, así como un difusor de los atractivos naturales y culturales que existen a lo largo y ancho de todo el estado.

En ese sentido el turismo se caracteriza por diversificarse en varios segmentos, e.g (Turismo Náutico y Deportivo; el Turismo de Salud; Turismo de Naturaleza; Turismo Cultural; Turismo Especializado, y especialmente el Turismo de Reuniones) siendo este último el que organiza eventos y selecciona un destino para la realización de los mismos ya sea de un congreso, una convención, un viaje de incentivos o una feria o exposición independientemente de la existencia de crisis económicas y dejando una derrama económica en los sitios donde se lleva a cabo (Comunión, 2010).

Tanto el turismo como la producción del bacanora en el estado de Sonora, son industrias que actualmente se encuentran en desarrollo, y por lo tanto uniendo esfuerzos, se puede alcanzar un crecimiento económico, ya que el bacanora en la actualidad es un símbolo de identidad Sonorense que merece ser difundido para el marcado nacional e internacional.

En tal situación se ha detectado en la comunidad académica la falta de evidencias empíricas que aporten y desarrollen acciones para apoyar la difusión y sensibilización de la importancia del bacanora como un tema de identidad Sonorense que promueva un desarrollo económico y de promoción turística, de medir los impactos que generan realizar eventos especializado (como festivales) en torno al tema del bacanora, de la denominación de origen y conocer el perfil de quienes asisten a estos eventos, así como el grado de satisfacción de las actividades planeadas.

Por lo que se planean los siguientes cuestionamientos ¿Qué actividades académicas, culturales, infantiles, espectáculos y de negocios deben planearse en un evento especializado para generar identidad Sonorense? ¿Cuál es el género, la edad, el nivel de estudios y la procedencia de los visitantes al evento?

Bacanora Fest es un evento especializado que gira en torno al tema del Bacanora, el cual tiene una duración de 3 días e incluye actividades académicas, culturales, infantiles, espectáculos y de negocios. Surge como una respuesta a un estudio exploratorio a la necesidad de investigación, de formación, de mercadeo, de intercambio de ideas entre especialistas sobre el tema del Bacanora y su impulso para 
posicionar la identidad sonorense y promover el turismo principalmente el cultural y el de reuniones.

Dicho evento es de nueva creación y es un evento sin precedente en el estado de Sonora, el primero que se celebra en el Municipio de Rosario (Municipio con denominación de origen del Bacanora), el cual es uno de los municipios pioneros en incursionar en esta actividad.

Como es bien sabido los eventos turísticos se apoya en una serie de eventos especiales como fiestas, ferias, festivales, macro-exposiciones que conforman la dimensión no material de la identidad turística de una localidad (De la Calle Vaquero, 2002).

Un evento es aquel hecho presentado como resultado de una organización previa, cuya finalidad es reunir a un grupo de personas en un mismo tiempo, lugar, y espacio, los cuales comparten actividades para un mismo fin ya sea comercio, industria, motivos sociales, o cultura (Rosario Jijena, 2015).

Por otra parte "la organización de eventos es todo un proceso en el cual se planifica, prepara, y produce tomando como base la evaluación, definición, adquisición, asignación, dirección, control y análisis del tiempo, así como el presupuesto, las personas, los productos y los recursos para alcanzar los objetivos" (Silvers, EMBOK, 2004).

\section{Material y Método}

La metodología empleada tiene un enfoque mixto con alcance exploratorio y descriptivo del fenómeno de la industria del Bacanora y del turismo de reuniones, su diseño es no experimental de corte transversal, se empleó la metodología de planeación de eventos basada en la propuesta de Richero (2008).

\section{Muestra}

Se realizó un muestreo no probabilístico por conveniencia conformado por 410 sujetos, este tipo de muestreos se realizan cuando no se tiene acceso completo a la población de estudio (Hernández, Fernández y Baptista, 2006). El principal criterio fue que los 
participantes se registraran al evento programado Bacanora Fest Rosario y tuvieran la disposición de participar. La caracterización de la muestra se presenta en la Tabla 2.

\section{Tabla 2.}

Caracterización de la muestra

Características de la muestra

Género

Masculino

Femenino

$10-17$

$18-25$

26-33

$34-41$

$42-49$

$50-60$

60-75

No dijeron su edad

Nivel de Estudios

Primaria

Secundaria

Preparatoria

Licenciatura

Posgrado

Sin respuesta

Procedencia de los asistentes al evento

$$
\text { Rosario (Locales) }
$$

Cajeme

129

Hermosillo (capital del estado)

Yécora

Otros Municipios del Estado de Sonora

Otros estados de la república mexicana (Sinaloa,

$$
\text { Michoacán Y nuevo león). }
$$

Extranjeros (Argentina, Colombia y Brasil)

\section{9}

118

38

41

52

45

29

48
84

$230 \quad 56$

$\%$

$\%$

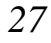

74

144

113

37

15
$10 \%$

$29 \%$

$9 \%$

$10 \%$

$13 \%$

$11 \%$

$7 \%$

$12 \%$ 
Sin embargo, cabe hacer mención que la afluencia de personas en el destino por motivo del evento del Bacanora Fest fue de más de 1000 visitantes al día durante los 3 días.

\section{Instrumentos}

Para obtener los datos se utilizó un instrumento de registro de asistencia al evento Bacanora Fest Rosario, que contempla variables sociodemográficas (i.e., genero, edad, nivel de estudios, procedencia de los asistentes al evento) para conocer las características sociales que describen a la población asistente utilizando una unidad de medida de categorías por rango (RAE, 2001).

Otro instrumento utilizado para la planeación del evento fue adaptado de (Richero, 2008) que contempla 5 fases.

1. Toma de decisiones preliminares: abarca la definición del tema, las metas y los objetivos, el perfil de los participantes y oradores, el diseño del evento.

2. Tareas de Avance: Incluyen la preparación de un presupuesto tentativo, el contacto y compromiso de los oradores y panelistas, así como la contratación de instalaciones, arreglos de traslados etc.

3. Promoción y contacto con los participantes. Establecer el vínculo e identificar a los posibles participantes al evento para lograr la meta.

4. Ejecución del evento: tareas, actividades complementarias.

5. Seguimiento y Evaluación. Se da seguimiento las actividades planeadas para verificar el cumplimiento de los objetivos, metas del evento, así mismo es recomendable documentar las mejores experiencias para detectar los programas de apoyo y mejora.

Por otro lado, se definieron las dimensiones para la planeación del programa de actividades para la ejecución del evento denominado Bacanora Fest Rosario contemplando 5 principales (ver tabla 3 ). 
Tabla 3.

Operacionalización de las actividades principales del Bacanora Fest

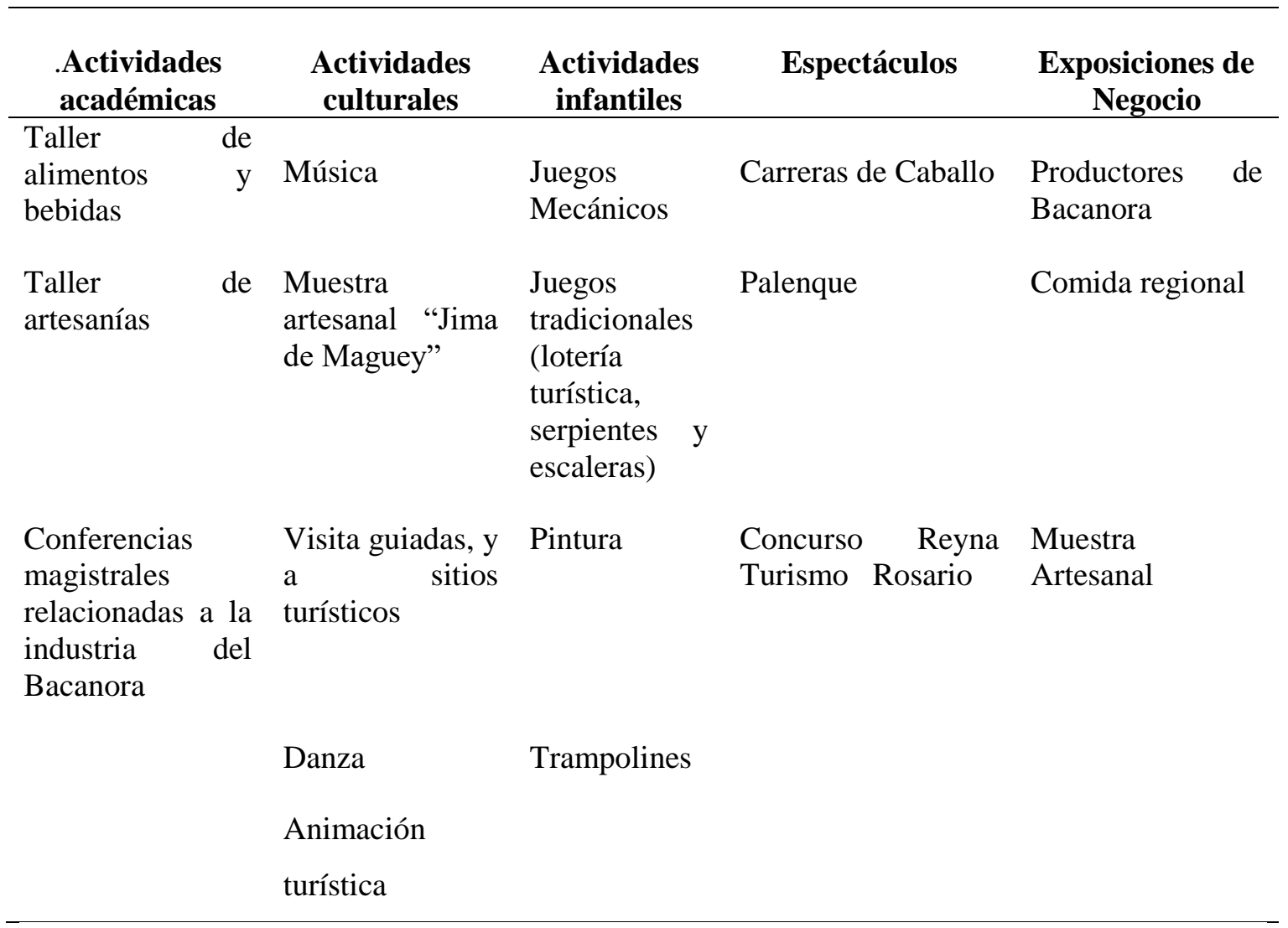

Fuente: Elaboración propia basada en los estudios de Cordero 2010.

\section{Resultados}

\section{ETAPA DE PREPARACION}

El evento surge como una respuesta a la necesidad de investigación, de formación, de mercadeo, de intercambio de ideas entre especialistas sobre el tema del Bacanora y su impulso para promover el turismo al ser parte de una identidad Sonorense. 
Tabla 4.

Etapa de preparación del primer festival

Toma de decisiones preliminares

Elección del tema del evento

Definición de metas y objetivos
Decisión sobre aspectos temáticos

\section{Objetivo General}

\section{"Bacanora Fest"}

Fomentar espacios académicos, artísticos y culturales de discusión y debate acerca del Bacanora para impulsar el desarrollo económico del Municipio de Rosario.

\section{Objetivos específicos}

1. Establecer este festival como evento continuo anual del Municipio de Rosario para generar identidad.

2. Apoyar a los productores del Municipio de Rosario el derecho para la protección a los procesos de fabricación y materias primas del producto de bacanora bajo la norma, al tener denominación de origen.

3. Apoyar la producción artesanal ya representan una fuente de ingresos para sus familias.

4. Difundir en la comunidad Rosarense la importancia de la denominación de origen de la producción de Bacanora.

5. Promover el turismo e impulsar la industria del Bacanora $y$ sus derivados.

6. Aumentar la visita de turistas y con ello el crecimiento económico al Municipio de Rosario.

La Industria del Bacanora

Aprovechamiento de los residuos de maguey

Taller de coctelería

Alimentos preparados con Bacanora

Exposición de arte mukimono con la temática del Bacanora.

Montaje de Vinata

Concurso de Jima de maguey

Bacanoreando por las calles de Tesopaco

"Animación turística y sociocultural" 
Los Asistentes: Determinación del Tipo y número de estos

Los Ponentes y conferencistas que están incluidos en el programa

\section{Selección de la fecha de realización y duración del evento}

\section{Selección del lugar del evento}

Elección de los patrocinadores y/o socios del proyecto

\section{Designación de un Coordinador General Designación de responsables de Comité y Organizadores}

El evento va dirigido principalmente a las familias del Sur de Sonora, Investigadores, académicos, estudiantes, empresarios, interesados en el estudio del tema del bacanora con una afluencia aproximada de 1000 asistentes durante los tres días.

Investigadoras de ITSON

Miembros del consejo Sonorense regulador del Bacanora

Universidades regionales ITSON, VIZCAYA, ITESCA

Investigadores y estudiantes de las áreas de biotecnología, turismo, gastronomía y administración

Productores regionales de Bacanora

Artesanos y Productos Regionales

Consejo Regulador del Bacanora

Instituto Sonorense de Cultura

Grupo Achai

Certamen Reyna Turismo Sonora

Misiones Culturales BJ y Rosario

(segunda Semana de Marzo, 3 dias)

Rosario Tesopaco, al ser un Municipio que tiene la denominación de origen y que cuenta con la facilidad de acceso al lugar e instalaciones, la comodidad, los servicios y el equipo. Apoyándonos con hoteles de cd. Obregón por la capacidad de hospedaje presente en el Municipio.

Secretaria de Economía

Comisión del Fomento al Turismo (COFETUR)

Instituto Sonorense de Cultura

Vizcaya, Itesca, Faccineto, Renault, Grupo

Soles, Arcelor Mittal, Productores de

Bacanora y Comercio Local de Rosario

Coordinadora General del Bacanora Fest

Responsable del Comité de Publicidad

Responsable del Comité de Seguridad

Responsables del Comité de Atención

Responsables del Comité de Catering

Responsable del Comité de Registro 
Responsable del Comité de Animación

Turística

Responsable del Comité de Control

Interno

Responsable del Comité de Logística

Responsable del Comité de Investigación

Preparación de los costos estimados

Presupuesto de cada comité patrocinadores

Fuente: Elaboración propia basada en los estudios de Cordero 2010.

\section{ETAPA DE EJECUCION}

Tabla 5

Actividades principales ejecutadas del Bacanora Fest

\begin{tabular}{|c|c|c|c|c|}
\hline $\begin{array}{l}\text { Actividades } \\
\text { académicas }\end{array}$ & $\begin{array}{l}\text { Actividades } \\
\text { culturales }\end{array}$ & $\begin{array}{c}\text { Actividades } \\
\text { infantiles }\end{array}$ & Espectáculos & $\begin{array}{c}\text { Exposiciones } \\
\text { de Negocio }\end{array}$ \\
\hline $\begin{array}{lr}\text { Taller } & \text { de } \\
\text { alimentos } & \mathrm{y} \\
\text { bebidas } & \end{array}$ & Música & $\begin{array}{l}\text { Juegos } \\
\text { Mecánicos }\end{array}$ & $\begin{array}{l}\text { Carreras } \\
\text { Caballo }\end{array}$ & $\begin{array}{ll}\text { Productores de } \\
\text { Bacanora }\end{array}$ \\
\hline $\begin{array}{l}\text { Taller } \\
\text { artesanías }\end{array}$ & $\begin{array}{l}\text { Muestra } \\
\text { artesanal "Jima } \\
\text { de Maguey" }\end{array}$ & $\begin{array}{l}\text { Juegos } \\
\text { tradicionales } \\
\text { (lotería } \\
\text { turística, } \\
\text { serpientes y } \\
\text { escaleras) }\end{array}$ & Palenque & $\begin{array}{l}\text { Comida } \\
\text { regional }\end{array}$ \\
\hline \multirow{2}{*}{$\begin{array}{l}\text { Conferencias } \\
\text { magistrales } \\
\text { relacionadas a } \\
\text { la industria del } \\
\text { Bacanora }\end{array}$} & $\begin{array}{l}\text { Visita guiadas, } \\
\text { y a sitios } \\
\text { turísticos }\end{array}$ & Pintura & $\begin{array}{l}\text { Concurso } \\
\text { Reyna } \\
\text { Turismo } \\
\text { Rosario }\end{array}$ & $\begin{array}{l}\text { Muestra } \\
\text { Artesanal }\end{array}$ \\
\hline & $\begin{array}{l}\text { Danza } \\
\text { Animación } \\
\text { turística } \\
\text { Mixologia con } \\
\text { bacanora }\end{array}$ & Trampolines & & \\
\hline
\end{tabular}

Fuente: Elaboración propia basada en los estudios de Cordero 2010. 


\section{ETAPA DE EVALUACION Y SEGUIMIENTO}

En esta etapa se realizó un sondeo exploratorio entre 132 asistentes que participaron conocer las actividades que habían sido mayormente de su interés con las actividades planeadas y ejecutadas para evaluar las expectativas de los asistentes siendo las siguientes:

\section{Tabla 5}

Actividades mencionadas con mayor frecuencia de los que consumieron durante Bacanora Fest

\begin{tabular}{ll}
\hline Actividades & \\
\hline Programa completo & $97 \%$ \\
Bacanora y mixologia & $98 \%$ \\
Exposición de productos & $96 \%$ \\
Reyna Turismo & $90 \%$ \\
Bailes tradicionales & $87 \%$ \\
Seguridad & $90 \%$ \\
Hospitalidad & $98 \%$ \\
Animación turística del & $99 \%$ \\
recorrido con el burrito & \\
Convivencia y ambiente & $94 \%$ \\
familiar & \\
Conferencias & $93 \%$ \\
\hline
\end{tabular}

Fuente: elaboración propia

Por otro lado, los resultados del evento se estructuraron en 3 dimensiones para describir el fenómeno del turismo en esta actividad desarrollada.

Impacto Social y cultural: Se contó con una muestra de 410 asistentes registrados a las actividades de exposición, demostración, actividades culturales, conferencias y talleres.

Impacto económico: se contó con la participación de 48 stand que pusieron a la venta para los visitantes productos regionales del Municipio, como: Bacanora, puestos de comida regional, pan tradicional, chiltepín, machaca, tamales, artesanías, tejidos en palma.

En un escenario conservador se contabilizó un gasto promedio diario de $\$ 500$ por persona incluyendo transporte, hospedaje, alimentos, suvenir, contemplando 1000 visitantes diarios los tres días del evento se generó una derrama económica aproximadamente por la cantidad $\$ 1,500,000$ de (un millón quinientos mil pesos 00/100 M.N) que vino a beneficiar a las familias del Sur de Sonora principalmente al Municipio de Rosario. 


\section{Impacto académico:}

De los talleres con mayor demanda los asistentes prefirieron el taller de "Preparación de alimentos y bebidas con bacanora", seguido con la conferencia de Conferencia de "La Industria del Bacanora" lo cual se traduce en que a las personas les gusta conectar con los sentidos participando en actividades académicas prácticas.

Se contó con la formación de 37 estudiantes de Licenciatura en Administración de empresas turísticas y Licenciados en Administración de ITSON que participaron como organizadores del evento, así como 12 Investigadores de las áreas económicoadministrativas, tecnología de alimentos y biotecnología del ITSON.

Además, se contó con el apoyo de la Universidad Vizcaya de las Américas, Instituto Tecnológico Superior de Cajeme, así como estudiantes principalmente de Turismo, Gastronomía, Cultura y Biotecnología.

\section{Discusión}

El desarrollo de este tipo de eventos no solo fortalece la identidad, la imagen y el orgullo de un destino, sino que para el destino la realización de este tipo de eventos puede ser el atractivo principal de tal manera que supere algunos sitios naturales o culturales en la zona, así como las atracciones de carácter permanente que el destino ofrece para el visitante. Esta dinámica asegura un flujo y una provisión de empleos temporales asociados a los eventos que de otra manera un destino pequeño o una comunidad receptora no podría generar (Cordero, 2010).

En la literatura internacional revisada hace mención que los eventos también tienen problemas sociales como el consumo de alcohol, incremento de basura, congestionamiento vehicular incluso delincuencia, por lo que son aspectos de deben estudiarse para no caer en esas situaciones sino que el evento contemple aspectos para contrarrestar esas problemáticas que pudieran suscitarse en futuras ediciones, y proyectarlo como un evento que promueve oportunidades económicas, como una opción de esparcimiento y recreación familiar pues contempla actividades infantiles y para todas las edades. 


\section{Conclusiones}

Los eventos de turismo de reuniones como los festivales y las ferian poseen la capacidad de atracción a una amplia gama de visitantes permitiendo a una región promover su imagen, desarrollan el orgullo local y generar una derrama económica en las sedes.

Además de sus alcances teóricos, los resultados aquí expuestos poseen contenidos empíricos, la población que asiste es de todas las edades pues es importante seguir promoviendo actividades infantiles.

Por otro lado, la participación en las exposiciones de negocios con los productos regionales más alla de los derivados del bacanora se contempla un incremento de empleo en la comunidad, aunque sea temporal. Si lo que se busca es incrementar los beneficios para la comunidad local, es importante corroborar que los residentes locales sean precisamente quienes se estén favoreciendo directamente de dicho empleo.

Sin duda el tema del Bacanora representa una gran oportunidad para el desarrollo de la industria del turismo como una identidad Sonorense, que impulse y promueva el turismo cultural, el turismo de reuniones y el turismo rural atendiendo la problemática de escasa oferta de productos turísticos en el estado de Sonora y contribuya a las acciones de recreación y esparcimiento. Estas acciones permitieron visualizar las oportunidades para generar desarrollo económico y social en los destinos donde se realice, sin embargo, su proyección a futuro debe ser el mercado internacional para traer turismo, ya que el alcance de la iniciativa actualmente es local y regional,

La iniciativa que se incursiono en el Municipio de Rosario, ha contribuido a denotar en el sector primario la formalización y regularización de la producción del Bacanora, han mejorado los procesos de producción, envasado y etiquetado basados en la NOM-168-SCFI-2004 ya que su proceso de producción actual es de forma artesanal. 
Sin embargo a raíz de su participación en el festival han mejorado la imagen para la comercialización de sus productos, han pensado en una marca, que se encuentran en proceso de registro, puesto que antes lo comercializaban en botellas de plástico o recicladas de algún envase de refresco; ahora han invertido en envases de vidrio que es la forma tradicional de hacer madurar la bebida (a decir de los productores) también gracias a esta iniciativa se hizo la invitación y se tuvo la oportunidad de participar con su producto en otros festivales por ejemplo el Séptimo Festival del Agave celebrado en el Estado de Guanajuato, en el cual el Bacanora y Sonora fueron los invitados cual tuvo muy buena aceptación.

El proyecto debe seguirse desarrollando involucrando a estudiantes e investigadores interesados en el tema a que colaboren, por un lado 1) para seguir generando propuestas y acciones que detonen el desarrollo de la industria del Bacanora respetando su forma tradicional de producción impactando en el desarrollo regional puesto que esto sería un gran valor agregado para fortalecer el desarrollo de la industria del turismo en Sonora. 2) Creación de nuevos productos Turísticos e.g.(Ruta del Bacanora, líneas de suvenires, parques temáticos, museos, paisajes agaveros, visitas guiadas, catas, mixología entre otros).

Su proyección debe ser como un símbolo de un legado y tradición, como un símbolo de identidad Sonorense.

Los sectores de la sociedad con los que se vinculó este proyecto fueron con el Gobierno estatal y Municipal, a través del consejo regulador del Bacanora y la dirección de desarrollo económico del Municipio, Universidades, familias del Sur de Sonora y de los 35 Municipios serranos del estado de Sonora que tienen denominación de origen, productores de Bacanora, Investigadores, académicos, estudiantes, empresarios, interesados en el estudio del tema del bacanora.

Sin duda alguna es de suma importancia continuar con el desarrollo de estas iniciativas que no solo traen beneficios económicos a la sede, sino también sociales al generar una identidad; el reto es grande, pero haciendo equipo entre instituciones académicas, gobierno, empresas se pueden logran impactos de esta naturaleza, ya que estas iniciativas también prospectan futuros emprendimientos e inversiones entre los interesados y como consecuencia desarrollo regional. 


\section{Agradecimientos}

En especial al programa para el Desarrollo Profesional Docente PRODEP de la Secretaria de Educación Pública (SEP) por el apoyo brindado.

Así mismo al programa de fomento y apoyo a la investigación (PROFAPI) 2020 atraves del Instituto Tecnológico de Sonora (ITSON).

Al H. Ayuntamiento de Rosario por las oportunidades brindadas y la colaboración para la realización de este festival.

Al Consejo regulador del Bacanora del Estado de Sonora, por su apoyo y difusión.

A la Comisión de Fomento al Turismo del estado de Sonora, por su apoyo y difusión.

Al canal de YouTube Turismo con Pao por su cobertura para cubrir el evento y su difusión.

A los estudiantes de Licenciatura de ITSON, es especial de las áreas de Turismo, Administración, Diseño Gráfico, Economía y finanzas, Biotecnología y contador público que colaboraron en servicio social contribuyendo en este proyecto.

A las Universidades de la Región del Sur de Sonora UVA (Universidad Vizcaya de las Américas) ITESCA (Instituto Tecnológico Superior de Cajeme) por su colaboración.

A los productores de Bacanora de los Municipios Rosario Tesopaco, Álamos, Bacanora, Yecora de la sierra del estado de Sonora, por atender la invitación al festival y por su participación en el mismo, así como por las facilidades brindadas para el trabajo de campo.

A los visitantes al Bacanora Fest por su cooperación y participación. 


\section{Referencias:}

Álvarez-Ainza M L, K A Zamora-Quiñonez, E Acedo-Félix, "Perspectivas para el uso de levaduras nativas durante la elaboración de bacanora". Revista Latinoamericana de Microbiología, 2009.

Arreglo de Lisboa relativo a la protección de las denominaciones de origen y su registro internacional recuperado el 20 de Mayo de 2021 en: https://www.wipo.int/export/sites/www/treaties/es/documents/pdf/lisbon.pdf

Asociación Internacional de Congresos y Convenciones (ICCA), recuperado el 06 de Diciembre del 2020 en : https://www.iccaworld.org/

Bercial, R. Á. \& Timón, d. A. B. (2005). Nuevas tendencias en el desarrollo de destinos turísticos: marcos conceptuales y operativos para su planificación y gestión. Cuadernos de turismo, 15, 27-43.

Cano, B. A. T., del Carmen Ayala Garza, R., \& del Campo, D. M. H. F. (2010). UNA NUEVA FORMA DE VENDER PARAISOS: TURISMO DE REUNIONES. TURyDES, 3(7).

Centro de Estudios Superiores de Turismo (CESTUR) recuperado el 05 de Diciembre del 2019 en: https://universidadesdemexico.mx/universidades/instituto-deestudios-superiores-de-turismo

Chen, Ch. Y Soo, K. (2007) Cost structure and productivity growth of the Taiwanese international tourist hotels, Tourism Management 28 (6), 1400-1407.

Comunión, t. Y. D. E. (2010). Una nueva forma de vender paraísos: turismo de reuniones. Trabajo, 3(7).

Consejo Sonorense Regulador de Bacanora (CSRB), recuperado el 05 de Diciembre del 2020 en : https://www.csrb.org.mx/

Cordero, J. C. M., Ontiveros, M. M. M., \& Guerrero, A. L. (2010). Impactos sociales de la feria internacional del caballo en México. Una perspectiva local. Estudios y perspectivas en turismo, 19(6), 1090-1104.

De la Calle Vaquero, M. (2002) "La ciudad histórica como destino turístico". Ariel, Barcelona.

Diario Oficial de la Federación," Solicitud de declaración general de protección de la denominación de origen del bacanora", 2000.

Gobierno del Estado de Sonora, Boletín Oficial del Gobierno del Estado de Sonora, número 51, Sección I, 1992

HERNÁNDEZ, R. FERNÁNDEZ-COLLADO, C. y BAPTISTA, P. (2006). Metodología de la Investigación. (4a Ed). México: McGraw Hill.

Hopkins D., Armando (editor y traductor) "La relación de Philips Segesser, correspondencia familiar de un misionero en Sonora en el año de 1737”. Hermosillo, 1991.

Illsley Granich, C. "El Mezcal Papalote del Chilapan: una marca colectiva campesina"(2004). Recuperado el 05 de diciembre del 2020 en: http://www.raises.org/PFNM-documentos.htm 
Mancilla-Margalli, N., \& López, M. G. "Carbohidratos solubles en agua y patrones de estructura de fructanos de las especies Agave y Dasylirion “, Diario de la química agrícola y alimentaria, 2006.

Mercado, Salvador." Mercadotecnia programada", $3^{\text {a }}$ edición, Editorial Noriega Limusa, 2004.

M. L. Gutiérrez-Coronado, E. Acedo-Félix, A. I. Valenzuela-Quintanar. "Industria del Bacanora y su proceso de Elaboración". Ciencia y Tecnología Alimentaria, 2007.

NOM-168-SCTI-2004 Norma Oficial Mexicana, Bebidas alcohólicas-Bacanora especificaciones de elaboración, envasado y etiquetado. Diario oficial de la Federación. Recuperado el 05 de diciembre del 2019 en https://www.dof.gob.mx/

Núñez, L. "Estrategias para el desarrollo de la industria del bacanora en Sonora". CIAD, A. C., Gobierno del Estado de Sonora, CONACYT, Fundación Produce Sonora A.C. Hermosillo, Sonora, 2004.

Organización Mundial de la propiedad intelectual (OMPI, 2010). Recuperado de https://wipolex.wipo.int/es/treaties/textdetails/12586

Organización de las Naciones Unidas para la Alimentación y Agricultura (FAO), recuperado el 11 de Diciembre del 2020 en: http://www.fao.org/3/x2919s/x2919s05.htm

Richero Alicia (2008) Planeación y ejecución de de eventos presenciales y en linea Editorial Trillas.

Rosario Jijena Sánchez, "Eventos, como organizarlos con éxito", 2 da. Edición, Ugerman Editor, 2015.

Secretaría de Economía del Gobierno de México Denominaciones de Origen \#OrgulloDeMéxico Consultado el 15 de abril de 2021 en https://www.gob.mx/se/articulos/denominaciones-de-origen-orgullodemexico

Secretaria de Turismo (SECTUR), recuperado el 04 de Abril de 2020 en: https://www.gob.mx/sectur/

Silvers, J, EMBOK. Event Management Body of Knowledge Project, recuperado el 13 de Diciembre del 2020 en : www.juliasilvers.com/embok.htm

Ulloa N., Pedro. (1910) "El estado de Sonora y su situación económica al aproximarse el primer centenario de la independencia nacional”. Hermosillo: Imprenta del Gobierno. 1993.

Vidal Salazar Solano, "La industria del bacanora: historia y tradición de resistencia en la sierra sonorense”, Región y Sociedad Vol.19, Hermosillo, 2007.

Cómo citar este artículo:

CLARK-MENDIVIL, Y., Esparza García, I. ., \& Rodríguez García, M. . (2021). Bacanora: Una bebida regional con denominación de origen que impulsa el turismo de reuniones. Revista De Investigación Académica Sin Frontera: División De Ciencias Económicas Y Sociales, (35), 23. https://doi.org/10.46589/rdiasf.vi35.393

https://revistainvestigacionacademicasinfrontera.unison.mx/index.php/RDIASF/article/view/393 


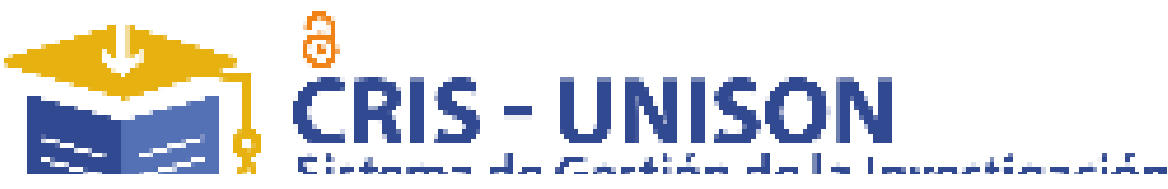
- Dialnet
latindex
If. IIIFACTOR
REDIB

Q: CiteFactor

is

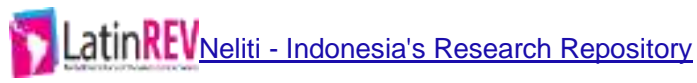

Donerandemion

DORA

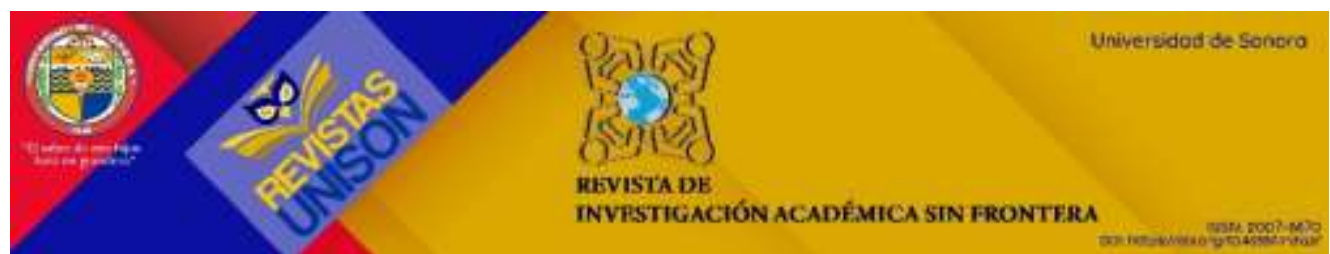

